Pacific Journal of Mathematics

COMMUTATOR GROUPS OF MONOMIAL GROUPS 


\title{
COMMUTATOR GROUPS OF MONOMIAL GROUPS
}

\author{
C. V. Holmes
}

This paper is a study of the commutator groups of certain generalized permutation groups called complete monomial groups. In [2] Ore has shown that every element of the infinite permutation group is itsself a commutator of this group. Here it is shown that every element of the infinite complete monomial group is the product of at most two commutators of the infinite complete monomial group. The commutator subgroup of the infinite complete monomial group is itself, as is the case in the infinite symmetric group, [2]. The derived series is determined for a wide class of monomial groups.

Let $H$ be an arbitrary group, and $S$ a set of order $B, B \geqq d, d=\boldsymbol{\aleph}_{0}$. Then one obtains a monomial group after the manner described in [1]. A monomial substitution over $H$ is a linear transformation mapping each element $x$ of $S$ in a one-to-one manner onto some element of $S$ multiplied by an element $h$ of $H$, the multiplication being formal. The element $h$ is termed a factor of the substitution. If substitution $u$ maps $x_{i}$ into $h_{j} x_{j}$, while substitution $v$ maps $x_{j}$ into $h_{t} x_{t}$, then the substitution $u v$ maps $x_{i}$ into $h_{j} h_{t} x_{t}$. A substitution all of whose factor are the identity $e$ of $H$ is called a permutation and the set of all permutations is a subgroup which is isomorphic to the symmetric group on $B$ objects. A substitution which maps each element of $S$ into itself multiplied by an element of $H$ is called a multiplication. The set of all multiplications form a subgroup which is the strong direct product of groups $H_{\alpha}$, each $H_{\alpha}$ isomorphic to $H$. Hereafter monomial substitutions which are permutations will be denoted by $s$, while those that are multiplications will be denoted by $v$. The monomial group whose elements are the monomial substitutions, restricted by the definitions of $C$ and $D$ as given below, will be denoted by $\Sigma(H ; B, C, D)$, where the symbols in the name are to be interpreted as follows, $H$ the given arbitrary group, $B$ the order of the given set $S, C$ a cardinal number such that the number of nonidentity factors of any substitution of the group is less than $C, D$ a cardinal number such that the number of elements of $S$ being mapped into elements of $S$ distinct from themselves by any substitution of the group is less than $D$. In the event $C=D=B^{+}, B^{+}$the successor of $B$, the resulting monomial group is termed the complete monamial group generated by the given group $H$ and the given set $S$. $S(B, M), d \leqq M \leqq D$, will denote the subgroup of permutations which map fewer than $M$ elements of $S$ onto elements of $S$ distinct from themselves, while $V(B, N)$,

Received October 25, 1958, and in revised form August 27, 1959. 
$d \leqq N \leqq C$, will denote the subgroup of multiplications which have fewer than $N$ nonidentity factors. In particular $S(B, d)$ denotes the subgroup of finite permutations and $V(B, d)$ the subgroup consisting of those multiplications which have finitely many nonidentity factors. The concept of alternating as associated with permutation groups may be extended in an obvious manner to monomial groups. $A(B, d)$ will denote the alternating subgroup of the permutation group $S(B, d)$, while $\sum_{A}(H ; B, d, d)$ will denote the alternating subgroup of the monomial group $\sum(H ; B, d, d)$. Any substitution may be written as the product of a multiplication and a permutation. Hence we may write $\Sigma(H ; B, C, D)=$ $V(B, C) \cup S(B, D)$, where $\cup$ here and throughout will mean group generated by the set. $G^{\prime}$ will be used to denote the commutator subgroup of the group $G$.

THEOREM 1. The commutator subgroup $V^{\prime}(B, C), d \leqq C \leqq B^{+}$, of $V(B, C)$ is the set of all elements

$$
v^{\prime}=\left(h_{1}^{\prime}, h_{2}^{\prime}, h_{3}^{\prime}, \cdots\right), h_{i}^{\prime} \in H^{\prime},
$$

where there exists an integer $N$ such that each $h_{i}^{\prime}$ is the product of $N$ or fewer commutators of $H$.

Proof. The theorem follows from the fact that $V(B, C)$ is the strong direct product, each of whose summands is isomorphic to $H$, together with the remark following the lemma page 308 of [2].

THEOREM 2. The commutator subgroup $S^{\prime}(B, C), d<C \leqq B^{+}$, of $S(B, C)$ is $S(B, C)$. The commutator subgroup $S^{\prime}(B, d)$ of $S(B, d)$ is $A(B, d)$.

The proof is contained in [2].

THeOREM 3. The commutator subgroup $\Sigma^{\prime}(H ; B, d, d)$ of $\Sigma(H ; B, d, d)$ is $A(B, d) \cup V^{+}(B, d)$ where $V^{+}(B, d)$ is the set of all elements of $V(B, d)$ whose product of factors is a member of $H^{\prime}$.

Proof. By reason of Theorem 2 we have

$\Sigma^{\prime}(H ; B, d, d) \supset A(B, d)$, and that

$\Sigma^{\prime}(H ; B, d, d) \supset V^{+}(B, d)$

will now be demonstrated.

If $h_{i}$ is the only nonidentity factor of the multiplication $v_{i}$, then the commutator $v_{i} s v_{i}^{-1} s$, where $s=\left(x_{i}, x_{j}\right)$, is a multiplication whose only nonidentity factors are $h_{i}$ and $h_{i}^{-1}$. It then follows that any multiplication $v$ of $V^{+}(B, d)$ with $n$ nonidentity factors can be written as the product of $n+1$ multiplications, $n$ of which are of the type of the commutator 
described above, and the remaining member having as its only nonidentity factor the product of the factors of $v$. But the first $n$ members of the product belong to $\Sigma^{\prime}(H ; B, \mathrm{~d}, \mathrm{~d})$, while the other member of the product is an element of $V^{\prime}(B, d)$, by reason of Theorem 1 , and hence

$$
\Sigma^{\prime}(H ; B, d, d) \supset V^{+}(B, d) \text {, since } V^{\prime}(B, d) \subset \Sigma^{\prime}(H ; B, d, d) .
$$

Then

$$
\Sigma^{\prime}(H ; B, d, d) \supset V^{+}(B, d) \cup A(B, d) .
$$

Since $G / G^{\prime}$ is abelian for any group $G$, and $G^{\prime}$ is the smallest group for which this is true, to demonstrate that

$$
\sum(H ; B, d, d) / V^{+}(B, d) \cup A(B, d)
$$

is abelian will imply that

$$
\Sigma^{\prime}(H ; B, d, d) \subset V^{+}(B, d) \cup A(B, d),
$$

and the conclusion of the theorem will follow.

That $V^{+}(B, d) \supset V^{\prime}(B, d)$ follows from the definition of $V^{+}(B, d)$, and hence $V(B, d) / V^{+}(B, d)$ is abelian. Therefore any two multiplications commute $\bmod V^{+}(B, d) \cup A(B, d)$. Since $A(B, d)$ consists of all even permutations there are but two cosets of $A(B, d)$ in $S(B, d)$, namely, $A(B, d)$ and $\left(x_{1}, x_{2}\right) A(B, d)$. Thus any element of the factor group $\Sigma(H ; B, d, d) / V^{+}(B, d) \cup A(B, d)$ has one of the forms

$$
v\left[V^{+}(B, d) \cup A(B, d)\right]
$$

or

$$
v\left(x_{1}, x_{2}\right)\left[V^{+}(B, d) \cup A(B, d)\right], v \in V(B, d) .
$$

But $v\left(x_{1}, x_{2}\right) v^{-1}\left(x_{1}, x_{2}\right)$ is the commutator $\left(h_{1} h_{2}^{-1}, h_{2} h_{1}^{-1}, e, \cdots\right)$ which belongs to $V^{+}(B, d)$. That is, $\left(x_{1}, x_{2}\right)$ and $v$ commute $\bmod \left[V^{+}(B, d) \cup A(B, d)\right]$, and hence $\Sigma(H ; B, d, d) / V^{+}(B, d) \cup A(B, d)$ is abelian, which implies $\Sigma^{\prime}(H ; B, d, d) \subset V^{+}(B, d) \cup A(B, d)$, and we have

$$
\Sigma^{\prime}(H ; B, d, d)=V^{+}(B, d) \cup A(B, d) .
$$

The following theorem asserts that the derived series for $\Sigma(H ; B, d, d)$ consists of but two distinct terms.

Theorem 4. The commutator subgroup $\Sigma^{\prime \prime}(H ; B, d, d)$ of $\Sigma^{\prime}(H ; B, d, d)$ is $\Sigma^{\prime}(H ; B, d, d)$.

Proof. $A(B, d)=A^{\prime}(B, d)$, as was demonstrated in Theorem 7 of [2], and hence $\Sigma^{\prime \prime}(H ; B, d, d)$ contains $A(B, d)$. 
Consider elements $v_{1}$ and $v_{2}$ of $\Sigma^{\prime}(H ; B, d, d)$, where the factors of $v_{1}$ are all $e$ except the first two and they are inverses of one another, and the factors of $v_{2}$ are all $e$ except the first and third and they are inverses of one another. The commutator $v_{1} v_{2} v_{1}^{-1} v_{2}^{-1}$, which is an element of $\Sigma^{\prime \prime}(H ; B, d, d)$, has as its first factor a commutator of $H$ and all other factors $e$. It then follows that any element of $V^{\prime}(B, d)$ is the product of elements of $\Sigma^{\prime \prime}(H ; B, d, d)$ and hence is an element of $\Sigma^{\prime \prime}(H ; B, d, d)$. That is $\Sigma^{\prime \prime}(H ; B, d, d) \supset V^{\prime}(B, d)$. Then one can in the manner described in the first part of Theorem 3 write any element $v$ of $V^{+}(B, d)$ as the product of $n+1$ elements, each member of the product being an element of $\Sigma^{\prime \prime}(H ; B, d, d)$. That is $\Sigma^{\prime \prime}(H ; B, d, d)$ contains $V^{+}(B, d)$, and hence $\Sigma^{\prime \prime}(H ; B, d, d)$ contains $V^{+}(B, d) \cup A(B, d)=$ $\Sigma^{\prime}(H ; B, d, d)$.

\section{THEOREM 5. The commutator subgroup}

$$
\sum_{A}^{\prime}(H ; B, d, d) \text { of } \sum_{A}(H ; B, d, d) \text { is } V^{+}(B, d) \cup A(B, d) \text {. }
$$

This theorem together with Theorem 3 states that $\sum(H ; B, d, d)$ has for its commutator subgroup $\sum_{A}(H ; B, d, d)$. This is the analogue for monomial groups of the result Ore obtains for permutation groups in [2], and as stated in the second part of Theorem 2 .

Proof. We have

$$
\Sigma^{\prime}(H ; B, d, d) \subset \sum_{A}(H ; B, d, d) \subset \Sigma(H ; B, d, d),
$$

hence,

$$
\Sigma^{\prime \prime}(H ; B, d, d) \subset \Sigma_{A}^{\prime}(H ; B, d, d) \subset \Sigma^{\prime}(H ; B, d, d) .
$$

Then by reason of Theorem 4 ,

$$
\Sigma^{\prime}(H ; B, d, d)=\Sigma^{\prime \prime}(H ; B, d, d)=V^{+}(B, d) \cup A(B, d) .
$$

Hence $\sum_{A}^{\prime}(H ; B, d, d)=V^{+}(B, d) \cup A(B, d)$.

Theorem 6. The commutator subgroup $\Sigma^{\prime}(H ; B, C, D), d<C \leqq D \leqq B^{+}$, of $\Sigma(H ; B, C, D)$ is $\Sigma(H ; B, C, D)$.

This theorem is also an analogue of a result Ore obtains in [2] for permutation groups as stated in the first part of Theorem 2.

Proof. It is shown in [2] that the commutator subgroup $S^{\prime}(B, D)$ of $S(B, D)$ is $S(B, D)$. Hence $\Sigma^{\prime}(H ; B, C, D)$ contains $S(B, D)$. The conclusion of the theorem will then follow if it can be demonstrated that $\Sigma^{\prime}(H ; B, C, D) \supset V(B, C)$. Let

$$
s=\left(\cdots, x_{-1}, x_{0}, x_{1}, \cdots\right)
$$


and

$$
v=\left(\cdots, h_{-1}, h_{0}, h_{1}, \cdots\right)
$$

be elements of $\Sigma(H ; B, C, D)$. Then the commutator $s v s^{-1} v^{-1}$ an element of $\Sigma^{\prime}(H ; B, C, D)$ has the form

$$
\left(\cdots, h_{0} h_{-1}^{-1}, h_{1} h_{0}^{-1}, h_{2} h_{1}^{-1}, \cdots\right) .
$$

Let

$$
v_{c}=\left(\cdots, c_{-1}, c_{0}, c_{1}, \cdots\right)
$$

be an arbitrary element of $V(B, C)$, and consider the following set of equations.

$$
\cdots, h_{0} h_{-1}^{-1}=c_{-1}, h_{1} h_{0}^{-1}=c_{0}, h_{2} h_{1}^{-1}=c_{1}, \cdots
$$

This set of equations has solutions,

$$
h_{0}=c_{-1}, h_{-1}=e, h_{n}=c_{n-1} h_{n-1}, h_{-n}=\left[\prod_{i=2}^{n} c_{-i}\right]^{-1} .
$$

Then if the factors of $v$ be represented in terms of the factors of $v_{c}$ as indicated above, we see that

$$
s v s^{-1} v^{-1}=v_{c} \in \Sigma^{\prime}(H ; B, C, D),
$$

and hence $\Sigma^{\prime}(H ; B, C, D)$ contains $V(B, C)$, and therefore

$$
\Sigma(H ; B, C, D)=\Sigma^{\prime}(H ; B, C, D) \text {. }
$$

Corollary 1. Any element $u$ of $\Sigma(H ; B, C, D), d<C \leqq D \leqq B^{+}$, is the product of at most two commutators.

Proof. Every element of $S(B, D)$ is a commutator of $S(B, D)$, as was shown in [2]. Every element of $V(B, C)$ is a commutator of $\Sigma(H ; B, C, D)$, as was shown in Theorem 6 . Therefore any element of $\Sigma(H ; B, C, D)$ which is either a multiplication or a permutation is a commutator. But every element of $\Sigma(H ; B, C, D)$ maybe written as the product of a multiplication and a permutation and consequently may be written as the product of two commutators.

To see that the assertion that every element of $\Sigma(H ; B, C, D)$ is the product of at most two commutators is the strongest possible, suppose every element of $\Sigma(H ; B, C, D)$ is a commutator. Let

$$
u \in \Sigma(H ; B, d, d) \subset \Sigma(H ; B, C, D) \text {. }
$$

Then $u=u_{1} u_{2} u_{1}^{-1} u_{2}^{-1}, u_{1}$ and $u_{2}$ elements of $\Sigma(H ; B, C, D)$. But since $u$ belongs to $\Sigma(H ; B, d, d)$ we can choose a $u_{1}^{*}$ and $u_{2}^{*}$ in $\Sigma(H ; B, d, d)$ by 
causing $u_{1}$ and $u_{2}$ to become the map of $x_{i}$ into $e x_{i}$ except for those maps which yield the permutation and nonidentity factors of $u$. It then follows that $u$ is an element of $\Sigma^{\prime}(H ; B, d, d)$, and hence $\Sigma(H ; B, d, d)=$ $\Sigma^{\prime}(H ; B, d, d)$. But this is a contradiction to Theorem 3.

\section{REFERENCES}

1. Ore, Oystein, Theory of monomial groups, Trans, Amer. Math. Soc. 51 (1942).

2. Ore, Oystein, Some remarks on commutators, Proc. Amer. Math. Soc. 2 (1951).

UNIVERSITY OF KANSAS AND

San Diego State College 


\section{PACIFIC JOURNAL OF MATHEMATICS}

\section{EDITORS}

David GILbarg

Stanford University

Stanford, California

\section{F. H. BRowneLL}

University of Washington

Seattle 5, Washington

\section{A. L. Whiteman}

University of Southern California Los Angeles 7, California

\section{J. PAIGe}

University of California

Los Angeles 24, California

\section{ASSOCIATE EDITORS}
E. F. BECKENBACH
T. M. CHERRY
D. DERRY

\author{
E. HEWITT \\ A. HORN \\ L. NACHBIN
}

\author{
M. OHTSUKA \\ H. L. ROYDEN \\ M. M. SCHIFFER
}

E. SPANIER

E. G. STRAUS

F. WOLF

\section{SUPPORTING INSTITUTIONS}

\author{
UNIVERSITY OF BRITISH COLUMBIA \\ CALIFORNIA INSTITUTE OF TECHNOLOGY \\ UNIVERSITY OF CALIFORNIA \\ MONTANA STATE UNIVERSITY \\ UNIVERSITY OF NEVADA \\ NEW MEXICO STATE UNIVERSITY \\ OREGON STATE COLLEGE \\ UNIVERSITY OF OREGON \\ OSAKA UNIVERSITY \\ UNIVERSITY OF SOUTHERN CALIFORNIA
}

\author{
STANFORD UNIVERSITY \\ UNIVERSITY OF TOKYO \\ UNIVERSITY OF UTAH \\ WASHINGTON STATE COLLEGE \\ UNIVERSITY OF WASHINGTON \\ AMERICAN MATHEMATICAL SOCIETY \\ CALIFORNIA RESEARCH CORPORATION \\ HUGHES AIRCRAFT COMPANY \\ SPACE TECHNOLOGY LABORATORIES \\ NAVAL ORDNANCE TEST STATION
}

\footnotetext{
Mathematical papers intended for publication in the Pacific Journal of Mathematics should be typewritten (double spaced), and the author should keep a complete copy. Manuscripts may be sent to any one of the four editors. All other communications to the editors should be addressed to the managing editor, L. J. Paige at the University of California, Los Angeles 24, California.

50 reprints per author of each article are furnished free of charge; additional copies may be obtained at cost in multiples of 50 .
}

The Pacific Journal of Mathematics is published quarterly, in March, June, September, and December. The price per volume (4 numbers) is $\$ 12.00$; single issues, $\$ 3.50$. Back numbers are available. Special price to individual faculty members of supporting institutions and to individual members of the American Mathematical Society: $\$ 4.00$ per volume; single issues, $\$ 1.25$.

Subscriptions, orders for back numbers, and changes of address should be sent to Pacific Journal of Mathematics, 2120 Oxford Street, Berkeley 4, California.

Printed at Kokusai Bunken Insatsusha (International Academic Printing Co., Ltd.), No. 6, 2-chome, Fujimi-cho, Chiyoda-ku, Tokyo, Japan.

PUBLISHED BY PACIFIC JOURNAL OF MATHEMATICS, A NON-PROFIT CORPORATION

The Supporting Institutions listed above contribute to the cost of publication of this Journal, but they are not owners or publishers and have no responsibility for its content or policies. 


\section{Pacific Journal of Mathematics}

\section{Vol. 10, No. $4 \quad$ December, 1960}

M. Altman, An optimum cubically convergent iterative method of inverting a linear bounded operator in Hilbert space . . . . . . . . . . . . . . . . . . . . . . . . . . 1107

Nesmith Cornett Ankeny, Criterion for rth power residuacity ................. 1115

Julius Rubin Blum and David Lee Hanson, On invariant probability measures I . . . . . 1125

Frank Featherstone Bonsall, Positive operators compact in an auxiliary topology ..... 1131

Billy Joe Boyer, Summability of derived conjugate series . . . . . . . . . . . . . . . . 1139

Delmar L. Boyer, A note on a problem of Fuchs . . . . . . . . . . . . . . . . . 1147

Hans-Joachim Bremermann, The envelopes of holomorphy of tube domains in infinite

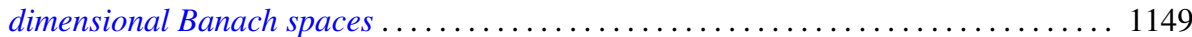

Andrew Michael Bruckner, Minimal superadditive extensions of superadditive

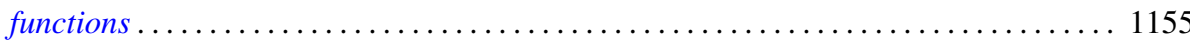

Billy Finney Bryant, On expansive homeomorphisms .................... 1163

Jean W. Butler, On complete and independent sets of operations in finite algebras . . . . . 1169

Lucien Le Cam, An approximation theorem for the Poisson binomial distribution ...... 1181

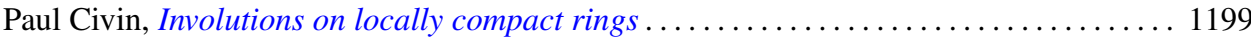

Earl A. Coddington, Normal extensions of formally normal operators . . . . . . . . . 1203

Jacob Feldman, Some classes of equivalent Gaussian processes on an interval ........ 1211

Shaul Foguel, Weak and strong convergence for Markov processes . . . . . . . . . . . 1221

Martin Fox, Some zero sum two-person games with moves in the unit interval ........ 1235

Robert Pertsch Gilbert, Singularities of three-dimensional harmonic functions . . . . . . . 1243

Branko Grünbaum, Partitions of mass-distributions and of convex bodies by

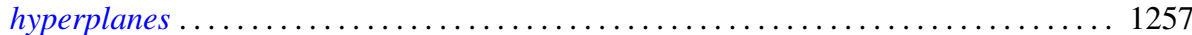

Sidney Morris Harmon, Regular covering surfaces of Riemann surfaces ........... 1263

Edwin Hewitt and Herbert S. Zuckerman, The multiplicative semigroup of integers

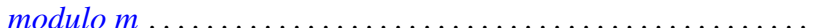

Paul Daniel Hill, Relation of a direct limit group to associated vector groups . ......... 1309

Calvin Virgil Holmes, Commutator groups of monomial groups . .

James Fredrik Jakobsen and W. R. Utz, The non-existence of expansive homeomorphisms

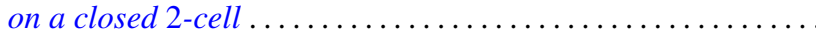

John William Jewett, Multiplication on classes of pseudo-analytic functions . . . . . . . 1323

Helmut Klingen, Analytic automorphisms of bounded symmetric complex domains . . . . 1327

Robert Jacob Koch, Ordered semigroups in partially ordered semigroups . . . . . . . . 1333

Marvin David Marcus and N. A. Khan, On a commutator result of Taussky and

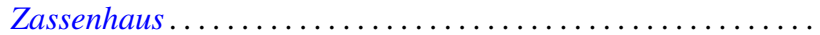

John Glen Marica and Steve Jerome Bryant, Unary algebras......

Edward Peter Merkes and W. T. Scott, On univalence of a continued fraction . . . . . . . 1361

Shu-Teh Chen Moy, Asymptotic properties of derivatives of stationary measures . . . . . 1371

John William Neuberger, Concerning boundary value problems . . . . . . . . . . . 1385

Edward C. Posner, Integral closure of differential rings . . . . . . . . . . . . . . . . . 1393

Marian Reichaw-Reichbach, Some theorems on mappings onto . . . . . . . . . . . . . 1397

Marvin Rosenblum and Harold Widom, Two extremal problems . . . . . . . . . . . . . . . . 1409

Morton Lincoln Slater and Herbert S. Wilf, A class of linear differential-difference

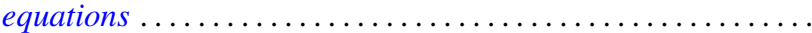

Charles Robson Storey, Jr., The structure of threads . . . . . . . . . . . . . . . . . . 1429

J. François Treves, An estimate for differential polynomials in $\partial / \partial z_{1},, \cdots, \partial / \partial z_{-} n \ldots \ldots 1447$

J. D. Weston, On the representation of operators by convolutions integrals . . . . . . . . 1453

James Victor Whittaker, Normal subgroups of some homeomorphism groups ......... 1469 